\title{
THE SOURCE OF MASS ACCRETED BY THE CENTRAL BLACK HOLE IN COOLING FLOW CLUSTERS
}

\author{
Noam Soker \\ Department of Physics, Technion-Israel Institute of Technology, Haifa 32000 Israel; \\ soker@physics.technion.ac.il.
}

\begin{abstract}
This paper reports the study of the cold-feedback heating in cooling flow clusters. In the cold-feedback model the mass accreted by the central black hole originates in non-linear over-dense blobs of gas residing in an extended region $(r \lesssim 5-30 \mathrm{kpc})$; these blobs are originally hot, but then cool faster than their environment and sink toward the center. The intra-cluster medium (ICM) entropy profile must be shallow for the blobs to reach the center as cold blobs. I build a toy model to explore the role of the entropy profile and the population of dense blobs in the cold-feedback mechanism. The mass accretion rate by the central black hole is determined by the cooling time of the ICM, the entropy profile, and the presence of inhomogeneities. The mass accretion rate determines the energy injected by the black hole back to the ICM. These active galactic nucleus (AGN) outbursts not only heat the ICM, but also change the entropy profile in the cluster and cause inhomogeneities that are the seeds of future dense blobs. Therefore, in addition to the ICM temperature (or energy), the ICM entropy profile and ICM inhomogeneities are also ingredients in the feedback mechanism.
\end{abstract}

Subject headings: cooling flows - galaxies: active - galaxies: clusters: general

\section{INTRODUCTION}

In recent years it has become clear that the intra-cluster medium (ICM) in cooling flow (CF) clusters of galaxies and CF galaxies must be heated, and the heating process should be stabilized by a feedback mechanism. (For recent papers and more references see Begelman \& Ruszkowski 2005; Buote et al. 2005; Donahue et al. 2005; Ensslin \& Vogt 2006; Fabian et al. 2005; Heinz \& Churazov 2005; Hoeft \& Bruggen 2004; Mathews et al. 2006; Nipoti \& Binney 2005; Omma \& Binney 2004; Ostriker \& Ciotti 2005; Vernaleo \& Reynolds 2006; Voit \& Donahue 2005; Peterson \& Fabian 2006; Pope et al. 2006; Brighenti \& Mathews 
2006.) As reported in most of these papers, the heating is based on active galactic nuclei (AGN) which do one or more of the following: launch jets into the ICM; inflate bubbles; cause turbulence; excite sound waves; and drive shocks. In some clusters weak shocks are detected, e.g., Perseus (Fabian et al. 2006), and a power of $\sim 2 \times 10^{43} \mathrm{erg} \mathrm{s}^{-1}$ in M87-Virgo (Forman et al. 2005), while in some cases strong shocks are driven to large distances in clusters, e.g., MS0735.6+7421 (McNamara et a. 2005), and a power of $\sim 10^{46} \mathrm{erg} \mathrm{s}^{-1}$ in Hercules A. (Nulsen et al. 2005). There are two questions regarding the AGN activity: (1) How is mass being fed to the central black hole (BH)?; and (2) How does feedback heating maintain stability? These questions are addressed in this paper.

Although heating substantially reduces the mass cooling rate, it seems it cannot completely suppress the CF (see review by Peterson \& Fabian 2006). The CF is defined as the process where mass near the cluster's center cools to low temperatures $\left(\lesssim 10^{4} \mathrm{~K}\right)$, and an inflowing still-hot gas replaces the cooling mass. Hicks \& Mushotzky (2005) argue that in $\sim 2 / 3$ of the $\mathrm{CF}$ clusters that they examined star formation could be the major sink for the cooling gas inferred from X-ray observations. In the CF cluster A1068, for example, there is an indication for star formation at a rate of $20-70 M_{\odot} \mathrm{yr}^{-1}$, about equal to the mass cooling rate in the central $\sim 30 \mathrm{kpc}$ inferred from X-ray observations (Wise et al. 2004; McNamara et al. 2004). In the CF cluster A2597, both extreme-UV and X-ray observations indicate a mass cooling rate of $\sim 100 M_{\odot} \mathrm{yr}^{-1}$, which is $\sim 0.2$ times the value quoted in the past based on ROSAT X-ray observations (Morris \& Fabian 2005). In the CF cluster A2029, Clarke et al. (2004) find a substantial amount of gas at a temperature of $10^{6} \mathrm{~K}$; a CF model gives a mass cooling rate of $\sim 50 M_{\odot} \mathrm{yr}^{-1}$. Salome \& Combes (2006) argue that the cold molecular gas in Perseus has its origin in the $\mathrm{CF}$ in this cluster. These observations suggest that in many clusters the $\mathrm{CF}$ is not completely suppressed, but rather occurs at a much lower rate, $\sim 5-20 \%$ of that in models with no heating.

The moderate CF model (Soker et al. 2001; Soker \& David 2003; Soker 2004) is one of the CF models where the mass cooling rate is low. The moderate $\mathrm{CF}$ model is different from many early proposed heating processes whose aim was to completely prevent the CF in clusters of galaxies. The main ingredient of the moderate CF model is that the effective age, i.e., the time period since the last major disturbance of the ICM inside the cooling radius $r_{c} \sim 100 \mathrm{kpc}$, is much shorter than the cluster age (e.g., Binney \& Tabor 1995; Binney 2004; Soker et al. 2001). The cooling radius is defined as the place where the radiative cooling time equals the cluster age. Heating in the moderate $\mathrm{CF}$ model was originally proposed to be intermittent with strong shocks (Soker et al. 2001), as observed in some cases (Nulsen et al. 2005; McNamara et a. 2005), but the basic idea holds for steady heating, or for heating in short intervals (Binney 2004). 
Pizzolato \& Soker (2005; hereafter PS05) study the feedback between heating and cooling of the ICM in the frame of the moderate CF model, adopting the mechanism in which a central BH accretes mass and launches jets and/or winds. PS05 propose that the feedback occurs within the entire cool inner region $(r \sim 5-30 \mathrm{kpc})$, where nonlinear over-dense blobs of gas with a density contrast $\rho / \rho_{a} \gtrsim 2$ cool fast and are removed from the ICM before experiencing the next major AGN heating event; $\rho$ is the density of a dense blob and $\rho_{a}$ the abient density. If the entropy profile is shallow, some of these blobs cool and sink toward the central $\mathrm{BH}$ hole, while others might form stars and cold molecular clouds. This scenario, where the BH is accreting cold gas, is termed cold feedback. This accretion process is different from the commonly assumed accretion mode in feedback models, where the BH accretes hot gas from its vicinity via a Bondi-type accretion flow (e.g. Churazov et al. 2002; Nulsen 2004; Omma \& Binney 2004; Chandran 2005). Soker \& Pizzolato (2005) propose that a large fraction of the gas cooling to low temperatures of $T<10^{4} \mathrm{~K}$ in $\mathrm{CF}$ clusters gains energy directly from the central $\mathrm{BH}$, (i.e., the accretion disk or jets close to the $\mathrm{BH}$ ). Most of this cool gas is accelerated to nonrelativistic high velocities, $v_{j} \simeq 10^{3}-10^{4} \mathrm{~km} \mathrm{~s}^{-1}$, after flowing through, or close to, an accretion disk around the central BH. Although massive outflows were not found yet in jets from AGNs in CF clusters, massive AGN-outflows are known to exist (e.g., Morganti et al. 2005).

PS05 find the conditions under which the dense blobs formed by perturbations might cool to low temperatures $\left(T<10^{4} \mathrm{~K}\right)$ and feed the BH to be as follows. (1) An overdense blob must be prevented from reaching an equilibrium position in the ICM; therefore, it has to cool fast, and the density, or more general the entropy, profile of the ambient gas should be shallow. (2) Nonlinear perturbations are required; they might have been chiefly formed by previous AGN activity. (3) The cooling time of these nonlinear perturbations should be shorter than several times the typical interval between successive AGN outbursts. (4) Thermal conduction around the blobs should be suppressed in order not to evaporate the blobs. This paper elaborates on the results of PS05 by constructing a simple toy model aim at discovering the nature of feedback heating and answering in part the two questions posed in the first paragraph of the Introduction regarding the source of the mass accreted by the $\mathrm{BH}$.

\section{THE MODEL}

I construct a toy CF cluster model as follows.

(1) Density profile. I assume the density profile to be of the form

$$
\rho=\rho_{0}\left(1+R^{2}\right)^{-1},
$$


where

$$
R \equiv \frac{r}{r_{s}},
$$

$r$ is the distance from the cluster center (radius), $\rho_{0}$ is the gas density at the center, and $r_{s}$ is the radius where the density falls to half its central value. This profile is simple, yet it keeps the main properties of the density profile in CF clusters-flat near the center and steep at $r \gtrsim r_{s} \simeq 3-30 \mathrm{kpc}$, depending on the cluster, e.g., $r_{s} \sim 5 \mathrm{kpc}$ in M87/Virgo (Ghizzardi et al. 2004), $r_{s} \sim 20 \mathrm{kpc}$ in Hydra A (David et al. 2001), $r_{s} \sim 25 \mathrm{kpc}$ in A2052 (Blanton et al. 2001), and $r_{s} \sim 30 \mathrm{kpc}$ in Perseus (Schmidt et al. 2002).

(2) Dense blobs. I follow PS05 and assume that the BH accretes dense cold blobs. These are formed from density perturbations scattered in the $\mathrm{CF}$ region. The density perturbations themselves can be a result of previous AGN outbursts, which are capable of disrupting the ICM (e.g., O'Sullivan et al. 2005). The falling of dense blobs through the ICM in CF clusters was discussed before, e.g., by Fabian (2003), who showed that such falling blobs can release gravitational energy and contribute to the heating of the ICM. The cold feedback model is different in that most of the heating comes from the accretion of these blobs by the central $\mathrm{BH}$. For simplicity I assume that the perturbation profile does not depend on the distance from the cluster center $r$. Namely, the fraction of mass residing in dense blobs cooling faster than their environment is $\delta_{0}$. As we'll see later, $\delta_{0}$ is an ingredient in the feedback process and it might depend on time.

(3) Mass cooling rate. The mass cooling rate of dense blobs in a shell with an inner radius $r$ and an outer radius $r+d r$ is

$$
d \dot{M}_{b}=\frac{4 \pi r^{2} \rho d r}{\tau_{\text {cool }}} \delta_{0},
$$

where the cooling time at radius $r$ is

$$
\tau_{\text {cool }}=\frac{5}{2} \frac{n k T}{\Lambda n_{e} n_{p}} \equiv K_{c} \frac{k T}{\rho} .
$$

The symbols have their usual meaning: $n_{e}, n_{p}$, and $n$ are the electron, proton, and total number density, respectively, $\Lambda n_{e} n_{p}$ is the cooling rate per unit volume, $k$ is the Boltzmann constant, $T$ is the temperature, and the last equality defines $K_{c}(T) \equiv(5 / 2) n \rho / \Lambda n_{e} n_{p}$. In the relevant temperatures for the inner regions of CF clusters $T \sim 2-5 \times 10^{7} \mathrm{~K}$, and the dependence of $K_{c}$ on $T$ is week. I therefore take $K_{c}$ to be a constant.

(4) The role of entropy profile. I assume a simple phenomenological relation between the number of dense blobs that cool and are then accreted by the BH and the total number of dense blobs at radius $r$. Following the results of PS05 (PS05 Fig. 9), the condition for 
accretion is that the entropy profile be shallow. Blobs that fall too fast don't have time to cool much faster than the environment they fall through; they reach an inner radius where they are in equilibrium, i.e., their entropy equals that of their new environment. As entropy profiles becomes steeper, more blobs will reach equilibrium and not fall to the center. Taking the entropy to be $K_{s}=k T n^{-2 / 3}$, the fraction of cooling blobs that fall all the way to the center is

$$
f=1-A \frac{d \ln K_{s}}{d \ln r}
$$

where $A \sim 1$ is a constant of the toy model, and if equation (5) gives $f<0$ I set $f=0$. For example, if the temperature is constant, then by equation (1)

$$
\frac{d \ln K_{s}}{d \ln r}=-\frac{2}{3} \frac{d \ln \rho}{d \ln r}=\frac{4}{3} R^{2}\left(1+R^{2}\right)^{-1}
$$

In that case $d \ln K_{s} / d \ln r=4 / 3$ for $r \gg r_{s}$, and I therefore take $A=3 / 4$, so that by substituting equation (6) in equation (5) one finds for a constant temperature

$$
f_{T}=\left(1+R^{2}\right)^{-1}
$$

(5) Total mass cooling rate. Combining equations (1) - (5) gives the BH mass accretion rate per unit radius (radial length) of mass originates at radius $r$

$$
\frac{d \dot{M}_{B H}}{d r}=\frac{4 \pi \delta_{0} \rho_{0}^{2} r_{s}^{2}}{K_{c} k T} \frac{R^{2}}{\left(1+R^{2}\right)^{2}} f
$$

(6) AGN power. Part of the cooling mass is accreted by the $\mathrm{BH}$, this part is parameterized by $\delta_{0}$; the other part being expelled back to the ICM (Soker \& Pizzolato 2005), or forms stars. I take the energy deposited by the central BH into the cluster to be

$$
L_{B H}=\eta c^{2} \dot{M}_{B H}
$$

where $c$ is the light speed.

\section{HEATING AND COOLING}

\subsection{Cooling}

The power emitted (in the X-ray) by gas residing inside a radius $R_{x}$ is given by

$$
L_{x}\left(R_{x}\right)=\int_{0}^{R_{x}} \Lambda n_{e} n_{p} 4 \pi r^{2} d r
$$


Substituting for the density from equation (1) gives

$$
L_{x}\left(R_{x}\right)=\frac{5}{2} \frac{4 \pi \rho_{0}^{2}}{\mu m_{H} K_{c}} r_{s}^{3} \int_{0}^{R_{x}} \frac{R^{2}}{\left(1+R^{2}\right)^{2}} d R=\frac{5}{2} \frac{4 \pi \rho_{0}^{2}}{\mu m_{H} K_{c}} r_{s}^{3} \frac{1}{2}\left[\frac{-R_{x}}{1+R_{x}^{2}}+\tan ^{-1} R_{x}\right],
$$

where $\mu m_{H}$ is the mean mass per particle. The cooling rate per unit radius in the model behaves as $d L_{x} / d R \propto R^{2} /\left(1+R^{2}\right)^{2}$; it increases from the center to $R=1$, and then decreases. This qualitatively matches observation in the relevant range (see fig. 8 of Voigt \& Fabian 2004). The cooling rate of the entire cluster is given by substituting $R_{x} \gg 1$ in equation

$$
L_{x}(\text { total })=\frac{5 \pi^{2}}{2} \frac{\rho_{0}^{2} r_{s}^{3}}{\mu m_{H} K_{c}}
$$

\subsection{Blobs Accretion Rate Per Unit Radial Length}

I consider now a case where the temperature is constant in the inner region. Substituting the constant temperature case $f=f_{T}$ (eq. 7) in equation (8) yields

$$
\frac{d \dot{M}_{B H-T}}{d R}=\frac{4 \pi \delta_{0} \rho_{0}^{2} r_{s}^{3}}{K_{c} k T} \frac{R^{2}}{\left(1+R^{2}\right)^{3}} .
$$

The mass accretion rate in units of $4 \pi \delta_{0} \rho_{0}^{2} r_{s}^{3} / K_{c} k T$ as given by equation (13) is plotted by the thick line in Figure 1. For comparison the rate when the entropy role in the model is not considered, namely $f=1$ in equation (8), is plotted by a dashed-dotted line. The entropy role in the toy model is to reduce the accretion rate mainly from large distances.

I consider two other temperature profiles in calculating the factor $f$ according to

$$
f_{g}=\frac{1}{\left(1+R^{2}\right)}-\frac{3}{4} \frac{d \ln T}{d \ln r}
$$

in line with equation (7). In the cluster A2052 (Blanton et al. 2001) the radius where the density falls to half its central value is $r_{s} \sim 25 \mathrm{kpc}$, and the temperature gradient can crudely be fitted by

$$
\frac{d \ln T}{d \ln R} \simeq 0 \quad(R \lesssim 0.6) ; \quad 1.3 \quad(0.6 \lesssim R \lesssim 1) ; \quad 0.2 \quad(1 \lesssim R \lesssim 4)
$$

where $R=1$ when $r=25 \mathrm{kpc}$ in this fit. The temperature, and as a result, the entropy sharply rise before density drops much. This substantially changes the entropy profile at $R \geq 0.6$. The mass accretion rate (per unit radial distance in units of $4 \pi \delta_{0} \rho_{0}^{2} r_{s}^{3} / K_{c} k T$ ) of dense blobs when $f=f_{g}$ with the temperature given by equation (15), is plotted by the thin 


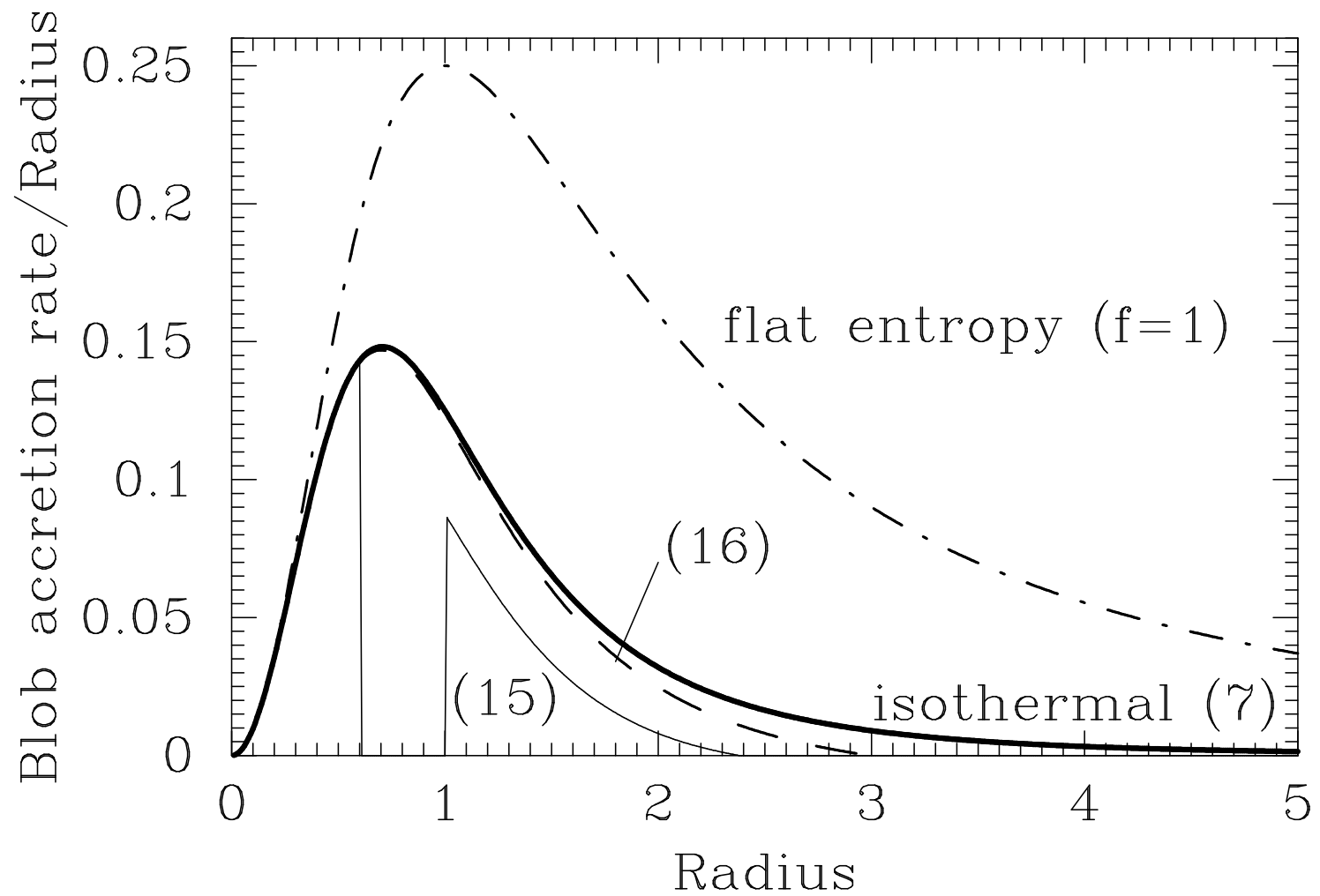

Fig. 1. - The mass accretion rate by the $\mathrm{BH}$ of cold blobs per unit radial length $R$ $\left(d \dot{M}_{B H} / d R\right)$ as given by equation (8). The radius is $R \equiv r / r_{s}$, where $r_{s}$ is the radius where density falls to half its value at the cluster center by equation (1). $d \dot{M}_{B H} / d R$ is plotted for different functions $f$ defined in equation (5), and in units of $4 \pi \delta_{0} \rho_{0}^{2} r_{s}^{3} / K_{c} k T$. The equation for $f$ for each line is given in parentheses. Solid thick line: equation (13), namely, for the isothermal case where $f=f_{T}$ (eq. 7). Dashed-dotted line: When the role of the entropy profile is neglected, $f=1$. Solid thin line: The value of $d \dot{M}_{B H} / d R$ for the case where $f$ is calculated from equation (14) by taking the temperature profile from equation (15). Dashed line: As the solid thin line, but the temperature gradient is given by equation (16). 
solid line in Figure 1. No accretion is expected to take place beyond $R=0.6$, despite the non zero value at $R>1$.

At the other extreme, in some clusters the temperature stays constant for large radii before rising. In M7/Virgo (Ghizzardi 2004) the temperature gradient can crudely be fitted by

$$
\frac{d \ln T}{d \ln R} \simeq 0.015 R^{2} \quad \text { for } \quad R \lesssim 4,
$$

where $R=1$ when $r=5 \mathrm{kpc}$ in this fit for M87/Virgo. The mass accretion rate (per unit radial distance in units of $4 \pi \delta_{0} \rho_{0}^{2} r_{s}^{3} / K_{c} k T$ ) of dense blobs when $f=f_{g}$ with the temperature given by equation (16), is plotted by the dashed line in Figure 1.

The temperature and density profiles are shallow near the center for all clusters, and as a consequence, the entropy profile is shallow there. The entropy then rises sharply (Voit \& Donahue 2005; Donahue et al. 2006). The temperature profiles used here represent the range of profiles found in $\mathrm{CF}$ clusters. In the majority of $\mathrm{CF}$ clusters, the entropy profile is almost flat near the center; thus for addressing the questions posed at this study it is sufficient to continue with the isothermal case.

\subsection{The Required Blobs Accretion Rate}

Equation (13) can be integrated to give the mass accretion rate originating in radii smaller than $R_{a}$

$$
\dot{M}_{B H-T}\left(R_{a}\right)=\int_{0}^{R_{a}} \frac{d \dot{M}_{B H}}{d R} d R=\frac{4 \pi \delta_{0} \rho_{0}^{2} r_{s}^{3}}{K_{c} k T} \frac{1}{8}\left[\frac{R_{a}^{3}-R_{a}}{\left(1+R_{a}^{2}\right)^{2}}+\tan ^{-1} R_{a}\right] .
$$

To compensate for cooling in the entire cluster, accretion should occur up to radius $R_{a}=R_{a t}$ which is found by equating $L_{x}$ (total) (eq. 12) with $L_{B H}$ (eq. 9), where the mass accretion rate from equation (17) is used in equation (9). This yields

$$
\left[\frac{R_{a t}^{3}-R_{a t}}{\left(1+R_{a t}^{2}\right)^{2}}+\tan ^{-1} R_{a t}\right]=\frac{3 \pi C_{s}^{2}}{\delta_{0} \eta c^{2}}=0.7\left(\frac{T}{3 \times 10^{7} \mathrm{~K}}\right)\left(\frac{\delta_{0} \eta}{10^{-4}}\right)^{-1},
$$

where $C_{s}=\left(5 k T / 3 \mu m_{H}\right)^{1 / 2} \simeq 820\left(T / 3 \times 10^{7} \mathrm{~K}\right)^{1 / 2} \mathrm{~km} \mathrm{~s}^{-1}$ is the ICM speed of sound. The scaling was chosen for a case where a fraction of $\delta_{0}=0.01$ of the mass is in dense blobs that reaches the $\mathrm{BH}$, and the energy deposited by the $\mathrm{BH}$ back to the ICM is a fraction of $\eta=0.01$ of the rest energy of the accreted mass. In Figure 2 the value of the radius $R_{a t}=r_{a t} / r_{s}$ as function $\delta_{0} \eta$ according to equation (18) is plotted. 


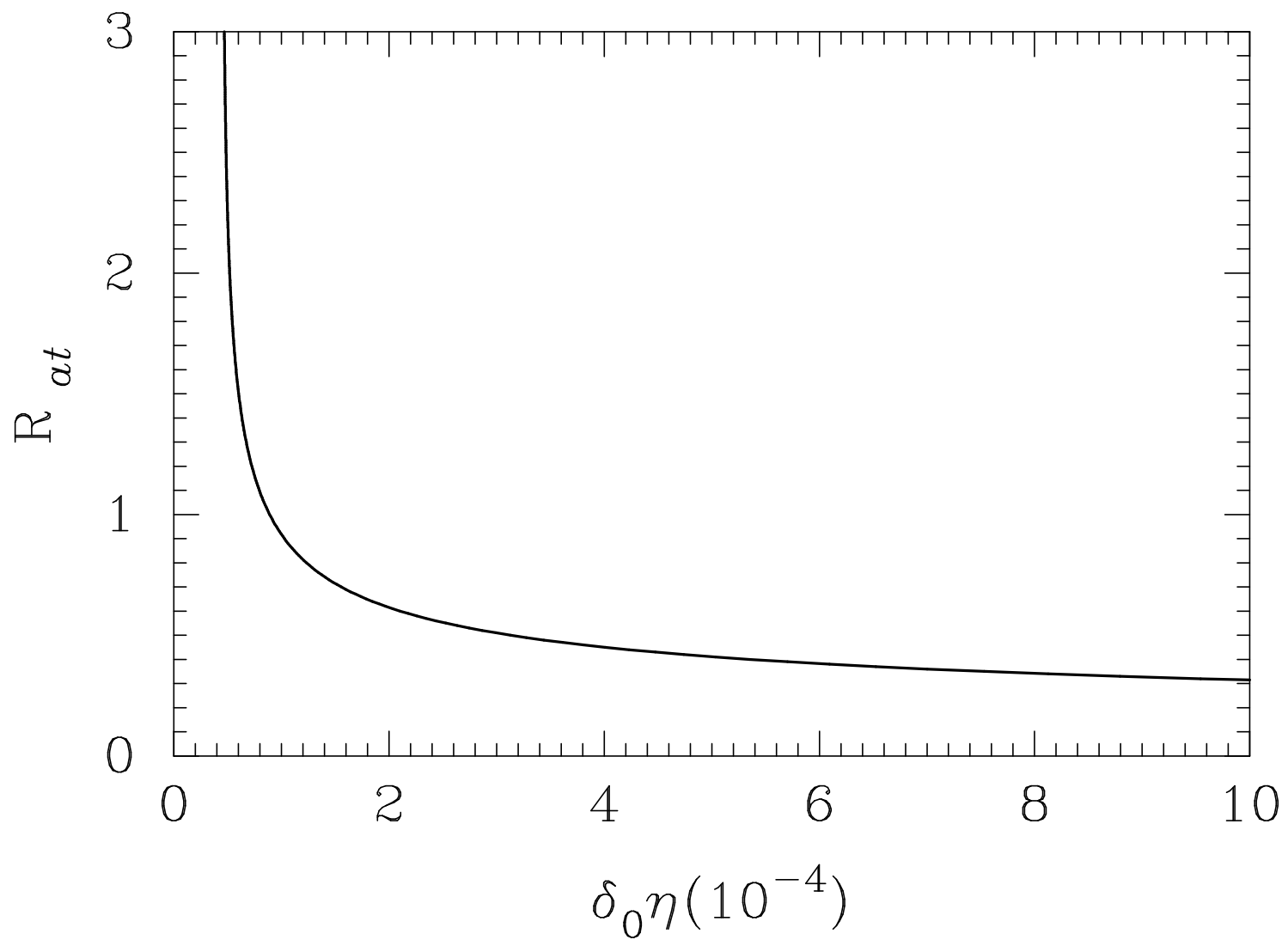

Fig. 2.- To heat the entire ICM in the toy model, accretion of dense blobs should occur from an extended region up to radius $R_{a t}$. The value of $R_{a t}$ is plotted as a function of the efficiency factor $\delta_{0} \eta$ as given by equation (18); $\delta_{0}$ is defined in equation (3) and $\eta$ is defined in equation (9). 


\section{THE FEEDBACK MECHANISM}

In Sections 2 and 3, the roles of the ICM entropy profile and the population of dense blobs were explored by a toy model. A cold blob can fall to the center of the cluster and be accreted by the central BH if the entropy profile near its origin is not too steep (PS05). If the entropy profile is steep, many sinking blobs will reach a region where the ambient entropy is equal to their entropy, and they stop sinking. A shallow entropy profile is a factor increasing the mass accretion rate. On the other hand, the entropy profile is influenced by AGN heating, which itself results from mass accretion. AGN heating of only the inner region makes entropy difference between the still cooling outer region and the heated inner region smaller (a shallower entropy profile). In the next outburst cycle, the accretion rate will be higher because of dense blobs falling in from regions further out. AGN heating of the entire ICM, on the other hand, makes the entropy profile in the $R \sim 1$ regions steeper. The conclusion is that the entropy profile is an ingredient in the feedback process, determining mainly the accretion rate from the region where most blobs reside in $R \sim 1$. The role of the entropy profile was parameterized by the function $f$ (eq. 5).

The dense cold blobs that are accreted by the central $\mathrm{BH}$ are formed in the inhomogeneous ICM. The inhomogeneities in the ICM result from previous AGN activities which form shocks, bubbles, and lift dense gas outward. This implies that the presence of inhomogeneities in the ICM is also an ingredient in the cold-feedback mechanism. The role of the inhomogeneous ICM was parameterized in the toy model by the factor $\delta_{0}$ (eq. 3 ).

The results of Section 3 suggest the following feedback mechanism in the frame of

the cold feedback scenario. For demonstrative purposes, the complicated feedback cycle is simplified by considering three basic types of time periods.

(P1) Between outbursts. The gas inside the CF region cools, with cold and dense blobs cooling first. They sink toward the center. Some of the cooling mass may form stars and cold clouds that are not accreted by the central BH. To reach the central BH, the entropy difference between the birth place of the blob and the center must be small. If not, the dense blob will reach a radius at which its entropy equals that of its surroundings.

(P2) Accretion with a weak outburst. If the entropy profile is shallow only in the very inner region, $R \lesssim 0.3$, then in accordance with equation (8) blobs primarily from this inner region are accreted; according to Figure 1 only a relatively small amount of mass is accreted. The AGN outburst is weak (depending on the efficiency factor $\delta_{0} \eta$ ) heating and disturbing only the very inner region. The outburst has three effects: (1) Increasing the cooling time of the inner region, thereby reducing the mass cooling rate from that region; (2) Increasing the entropy in the inner region, making the entropy profile shallower, allowing more dense 
blobs to be accreted from regions further out in the next cycle; and (3) Disturbing the inner region, forming density perturbations that are the seeds for dense blobs that will be accreted in the next cycle.

(P3) Strong outburst. After a weak AGN outburst, or several weak AGN outbursts, the entropy profile must become shallower because the region near $R \sim 1$ cools and its entropy decreases, while the heating of the inner region increases the entropy in the inner region. Because it was not heated efficiently by the weak outbursts, the region at $R \sim 1$ is cooler and its entropy is lower than previously. Both lower temperature and entropy ensure that more dense blobs from this region are accreted by the central BH. As seen from Figure 1 the region $R \sim 1$ could contribute large amount of mass. Figure 2 illustrates that if the perturbation fraction $\delta_{0}$ (eq. 3) and the heating efficiency $\eta$ (eq. 9 ) are such that $\delta_{0} \eta \gtrsim 10^{-4}$, then the accretion from the $R \sim 1$ region can heat the ICM in the entire cluster. The strong heating of the region up to the cooling radius and beyond (which extends to $R \gg 1$ in the toy model), increases the temperature and the cooling time. This reduce the mass cooling rate, and we are back to period $\mathrm{P} 1$.

The following comments are informative:

(1) The AGN outburst in CF clusters populate a continuous outburst energy distribution. It is only for demonstrative purposes that they were classified here 'weak' or 'strong'.

(2) If, in the toy model, the efficiency coefficient is $\delta_{0} \eta \lesssim 5 \times 10^{-5}$, then it is not possible to heat the entire cluster (Fig. 1$)$. The outer region $(R \sim 1-3)$ cools, the entropy profile becomes shallower, and the cooling time shorter. This implies that dense blobs with even smaller density contrast can be accreted by the $\mathrm{BH}$. This results in a larger value of $\delta_{0}$, and a stronger feedback heating. For the model to work, the value $\delta_{0} \eta$ must be able to increase to values $\gtrsim 10^{-4}$ as cooling proceeds. The population of dense blobs, as parameterized by $\delta_{0}$, is part of the feedback heating mechanism. This is an important additional ingredient to the assumed constant value of $\delta_{0}$ in the toy model.

(3) The entropy profile is also a significant ingredient in the feedback heating mechanism. Not only the cooling rate, but also the entropy profile, determine the mass accretion rate onto the central $\mathrm{BH}$, and consequently the heating rate.

(4) This cold-feedback cycle is based on the existence of a CF, but a moderate one (see Sec.

1). Parcels of gas cool to low temperatures, but the mass cooling rate is much smaller than in old CF models (Fabian 1994). 


\section{SUMMARY}

This paper discusses the cold-feedback mechanism (PS05). In the cold-feedback mechanism, the feedback occurs with the entire cool inner region $(r \lesssim 5-30 \mathrm{kpc})$, where non-linear over-dense blobs of gas with a large density contrast cool fast and are removed from the ICM before experiencing the next major AGN heating event. These blobs feed the BH at the center of the cluster. This process implies the presence of a cooling flow (CF), although a moderate one.

The feedback process was discussed in Section 4. Results are now incorporated into the frame of the moderate CF model by listing the ingredients of the feedback mechanism.

1. Energy Budget. The feedback is basically a heating-cooling feedback: cooling is primarily by radiative processes in the X-ray band; heating is by AGN activity, mainly jets launched by an accreting massive BH. Observations indicate that there is a substantial heating of the ICM, compensating for most, but not all, of the energy radiated in the $\mathrm{X}$-ray band. The heating rate is determined to a large extent by the central $\mathrm{BH}$ mass accretion rate. The main factor that determines the mass accretion rate is the cooling time of the ICM, which depends on the ICM density and on the ICM temperature. The ICM temperature (or the ICM energy per unit mass), is the main ingredient of the feedback mechanism. The cold-feedback mechanism involves other ingredients in maintaining the feedback stability. The other ingredients help to prevent both running heating to higher and higher temperatures and catastrophic cooling.

2. Dense cold blobs. In the cold-feedback model the source of the accreted mass are dense blobs resulting from inhomogeneities in the ICM. The ICM inhomogeneities result from the same AGN activity that heat the ICM (an AGN outburst forms the dense blobs that will be accreted in future cycles). The role of the inhomogeneous ICM was modeled in the toy model by the factor $\delta_{0}$ (eq. 3). If, in some regions, there is no heating for a long period of time, such as in the outer regions, the ICM cools with denser blobs cooling faster. The long period of no heating ensures also that blobs with a smaller density contrast have time to cool and increase their density contrast, resulting in a larger value of $\delta_{0}$; namely an increase in the dense blobs population that might be accreted by the BH. In the toy model heating efficiency is determined by the factor $\delta_{0} \eta$, where $\eta$ is the $\mathrm{BH}$ energy output efficiency (eq. 9). For a reasonable range

of $5 \times 10^{-5} \lesssim \delta_{0} \eta \lesssim 10^{-3}$ accretion from the region $R \lesssim 0.3-2$ can maintain the required accretion rate, as demonstrated in Figure 2.

3. Entropy profile. If the entropy profile is steep, many sinking blobs will reach a region where the ambient entropy equals their entropy, and therefore they stop sinking. Most 
relevant is the region $R \sim 1$, which can supply a large amount of mass to the BH (Fig. 1 ). A weak AGN outburst, heating only of the regions $R \lesssim 0.3$, makes the entropy profile in the region $R \sim 1$ shallower, increasing the mass accretion rate in the next cycle. Heating the entire ICM, up to regions $R \gg 1$, makes the entropy profile steeper in the region $R \sim 1$. The role of the entropy profile was parameterized by the function $f($ eq. 5$)$.

4. Mass cycle. Another possible, but not necessary, ingredient is a slow (few $\times 1000 \mathrm{~km} \mathrm{~s}^{-1}$ ) massive outflow (Soker \& Pizzolato 2005). This outflow starts in the accretion disk or its vicinity and carries a substantial fraction of the cold $\left(T \lesssim 10^{4} \mathrm{~K}\right)$ mass back to the ICM. This gas is shocked and heated to high temperatures. This outflow is significant to the mass cycle as well as to the energy cycle (Soker \& Pizzolato 2005). The mass cycle proposed by Soker \& Pizzolato (2005) is significantly different from the circulation flow proposed by Mathews et al. (2004), because Mathews et al. (2004) consider only hot gas with no cooling to temperatures below X-ray emission temperatures. In contrast to the moderate CF model adopted here, Mathews et al. (2004) do not consider the presence of a CF. The recent mass and energy cycle model of Brighenti \& Mathews (2006) is more similar to the model proposed by (Soker \& Pizzolato 2005) in that in contains some mass cooling to low temperatures.

The present results can be put in a more general perspective. The new X-ray observations by the XMM-Newton and Chandra Telescopes show the ICM in CF clusters to be highly inhomogeneous and to posses an almost flat entropy profiles near their centers. In the frame work of the cold-feedback scenario these observations support the presence of CFs in these clusters, although moderate CFs. Both the detection of gas at temperatures of $\sim 10^{6} \mathrm{~K}$ and the detection of star formation indicate the presence of $\mathrm{CF}$ in some clusters, but the more common inhomogeneities and flat entropy profiles also argue for the presence of CFs. The claim for a wide spread moderate CF phenomena is contrary to some claims, made during the past six years since these two X-ray telescopes were launched, supporting the absence of CFs.

This research was supported in part by the Asher Fund for Space Research at the Technion.

\section{REFERENCES}

Begelman, M. C., \& Ruszkowski, M. 2005, RSPTA, 363, 655 
Binney, J. 2004, in The Riddle of Cooling Flows in Galaxies and Clusters of Galaxies, ed. T. H. Reiprich, J. C. Kempner, \& N. Soker (Charlottesville: Univ. Virginia), http://www.astro.virginia.edu/coolflow/proc.php (astro-ph/0310222)

Binney, J., \& Tabor, G. 1995, MNRAS, 276, 663

Blanton, E. L., Sarazin, C. L., McNamara, B. R., \& Wise, M. W. 2001, ApJ, 558, L15

Brighenti, F., \& Mathews, W. G. 2006, ApJ, in press (astro-ph/06015)

Buote, D. A., Humphrey, P. J., \& Stocke, J. T., 2005, ApJ, 630, 750

Chandran, B. D. G. 2005, ApJ, 632,809

Churazov, E., Sunyaev, R., Forman, W., \& Böhringer, H. 2002, MNRAS, 332, 729

Clarke, T. E., Blanton, E. L., \& Sarazin, C. L. 2004, ApJ, 616, 178

David, L. P., Nulsen, P. E. J., McNamara, B. R., Forman, W., Jones, C., Ponman, T., Robertson, B., \& Wise, M. 2001, ApJ, 557, 546

Donahue, M., Horner, D. J., Cavagnolo, K. W., \& Voit, G. M. 2006, (astro-ph/0511401)

Donahue, M., Voit, G. M., O’Dea, C. P., Baum, S. A., \& Sparks, W. B. 2005, ApJ, 630, L13

Ensslin, T. A., \& Vogt, C. 2006, (astro-ph/0505517)

Fabian, A. C. 1994, ARA\&A, 32, 277

Fabian, A. C. 2003, MNRAS, 344, L27

Fabian, A. C., Reynolds, C. S., Taylor, G. B., \& Dunn, R. J. H. 2005, MNRAS, 363, 891

Fabian, A. C., Sanders, J. S., Taylor, G. B., Allen, S. W., Crawford, C. S., Johnstone, R. M., \& Iwasawa. K 2006, MNRAS, 366, 417

Forman, W. et al. 2005, ApJ, 635, 894

Ghizzardi, S., Molendi, S., Pizzolato, F., \& De Grandi, S. 2004, ApJ, 609, 638

Heinz, S., \& Churazov, E. 2005, ApJ, 634, L141

Hicks, A. K.; Mushotzky, R. 2005, ApJ, 635, L9

Hoeft, M., \& Bruggen, M. 2004, ApJ, 617, 896

Mathews, W. G., Brighenti, F., \& Buote, D. A. 2004, ApJ, 615, 662

Mathews, W. G., Faltenbacher, A., Brighenti, F. 2006, ApJ (astro-ph/0511151)

McNamara, B. R., Nulsen, P. E. J., Wise, M. W., Rafferty, D. A., Carilli, C., Sarazin, C. L., \& Blanton, E. L. 2005, Natur, 433, 45

McNamara, B. R., Wise, M. W., \& Murray, S. S. 2004, ApJ, 601, 173 
Morganti, R., Tadhunter, C. N., \& Oosterloo, T. A. 2005, A\&A, 444, L9

Morris, R. G., \& Fabian, A. C. 2005, MNRAS, 358, 585

Nipoti, C., \& Binney, J. 2005, MNRAS, 361, 428

Nulsen, P. 2004, in The Riddle of Cooling Flows in Galaxies and Clusters of Galaxies, ed. T. H. Reiprich, J. C. Kempner, \& N. Soker (Charlottesville: Univ. Virginia), http://www.astro.virginia.edu/coolflow/proc.php (astro-ph/0310195)

Nulsen, P. E. J., Hambrick, D. C., McNamara, B. R., Rafferty, D., Birzan, L., Wise, M. W., \& David, L. P. 2005, ApJ, 625, L9

Omma, H., \& Binney, J. 2004, MNRAS, 350, L13O

Ostriker, J. P., Ciotti, L. 2005, RSPTA, 363, 667O

O’Sullivan, E., Vrtilek, J. M., \& Kempner, J. C. 2005, ApJ, 624, L77

Peterson, J. R., \& Fabian, A. C. 2006, Physics Reports (astro-ph/0512549)

Pizzolato, F., \& Soker, N. 2005, ApJ, 632, 821 (PS05)

Pope, E. C. D., Pavlovski, G., Kaiser, C. R., \& Fangohr, H. 2006, MNRAS, (astro-ph/0601096)

Salome, P., \& Combes, F. 2006, SF2A-2005, eds. C. T. Contini, J-M. Hameury \& L. Pagani (eds) (EdP-Sciences) (astro-ph/0601097)

Schmidt, R. W., Fabian, A. C., \& Sanders, J. S. 2002, MNRAS, 337, 71

Soker, N. 2004, MNRAS, 350, 1015

Soker, N. \& David, L. P., 2003ApJ...589..770S

Soker, N., \& Pizzolato, F. 2005, ApJ, 622, 847

Soker, N., White, R, E., III, David, L. P., \& McNamara, B. R. 2001, ApJ, 549, 832

Vernaleo, J. C., \& Reynolds, C. S. 2006, sub. to ApJ (astro-ph/0511501)

Voigt, L. M., \& Fabian, A. C. 2004, MNRAS, 347, 1130

Voit, G. M., \& Donahue, M. 2005, ApJ, 634, 955

Wise, M. W., McNamara, B. R., \& Murray, S. S. 2004, ApJ, 601, 184 